\title{
Overcoming Challenges Across Production, Isolation and Antineoplastic Drug Loading of Exosomes
}

\author{
Esra Cansever Mutlu',2 \\ ${ }^{1}$ Beykent University \\ Faculty of Engineering and Architecture, Department of Biomedical \\ Engineering, Sarıyer, Istanbul, Turkey \\ esramutlu@ beykent.edu.tr \\ ${ }^{2}$ Bolu Abant Izzet Baysal University, BETUM, Golkoy Campus \\ Bolu, Turkey
}

\begin{abstract}
Exosome production and isolation seem the one of the latest, promising, highlight topic in Biomedical Engineering. Especially, "exosomes" is notable as the extracellular vehicles derived from many types of cells that provide vertical and horizontal transfer of genes, mRNAs, non-coding RNAs and proteins to their targets. Therefore, they have recently been used to targeted cancer therapy materials after loading these nucleic acids-based or synthetic drugs. Even if, nucleic acids-based loading to exosomes is most used, synthetic drug loading is the other its prominent role. However, the one of the big challenge is the not able to load desired amount of drug. In this talk, previously experienced exosomes of A549 ATCC® CCL-185 ${ }^{\mathrm{TM}}$ non-small lung cancer cell line and dexosomes of JAWSII ATCC ${ }^{\circledR}$ CRL-11904 ${ }^{\mathrm{TM}}$ immature dendritic cell line will be discussed. "in vitro" dexosome production restrictions and exosome isolation challenges will be shared. The talk likewise will be about their synthetic drug loading potential by ultrasonication technique which was implemented with Doxorubicin (DOX). Obtained results will be handled according to perspective of overcoming challenges during research of exosome as bio-based, tiny biomedical material in drug delivery.
\end{abstract}

Keywords: Exosome, Dexosome, Doxorubicin, Drug Loading, Ultrasonication.

\section{Introduction}

Exosomes represent new promising field for biomedical research. Exosomes has the tiny spherical shape as 40-150 nm in diameter and are the member of membrane enclosed structures called "Extracellular Vesicles" [1]. Exosomes can be isolated from the many cell types; such as, dendritic cells, cardiomyocytes, tumor cells, endothelial cells or reticulocytes, etc. Exosomes have different roles in sophisticated cell functions; such as, exchange molecular information, support physiological and pathological process, suppress immune surveillance, intercellular vertical and horizontal transfer of special proteins etc [2]. Last decades, therapeutic usage of exosomes has been emerging in the literature. The reason is that they have roles both as the potential bio-based targeting agent in cancer therapy and diagnose, tissue regeneration tool, immune response modulator and therapeutics for neurodegenerative diseases [3]. Moreover, since last decade, onset of standardization through production and isolation and further characterization of exosomes have been performing. It should be well-established according to environment of the cell source; such as, conditioned media in vitro or blood in vivo [4]. Nevertheless, low drug loading efficiency restriction after isolation can be encountered [5]. This talk will emphasize comparative challenges during production and isolation results of two different cell line exosomes A549 ATCC® CCL$185^{\mathrm{TM}}$ non-small lung cancer cell line and dexosomes of JAWSII ATCC ® CRL-11904 ${ }^{\mathrm{TM}}$ immature dendritic cell line [6, 7]. It will be emphasized overcoming DOX loading efficiency to dexosomes of ATCC ${ }^{\circledR}$ CRL-11904 ${ }^{\text {TM }}$ by ultrasonication.

\section{Results and Discussion}

Two different kind of exosome was produced by using two different cell lines. Production of ATCC ${ }^{\circledR}$ CRL-11904TM immature dendritic cell line performed and dexosomes were isolated by ultracentrifuge; similarly, in different times it was carried out from A549 ATCC® CCL-185 ${ }^{\mathrm{TM}}$ non-small lung cancer cell line to obtain their exosomes. Malvern Nanosizer and SEM images showed that exosomes A549 ATCC ${ }^{\circledR}$ CCL-185 ${ }^{\mathrm{TM}}$ non-small lung cancer cell line has larger diameter $\leq$ $200 \mathrm{~nm}$ and stability has $\leq-20 \mathrm{mV}$ (Fig.1C). Size result was mostly different from NTA measurements of them (Fig. 1A). 
Impurities from PBS effected experimental quality (Fig. 1B). DOX loading efficiency was just performed by using dexosomes of ATCC ${ }^{\circledR}$ CRL-11904 ${ }^{\mathrm{TM}}$ immature dendritic cell line. Results and encountered challenges will be presenting.

A

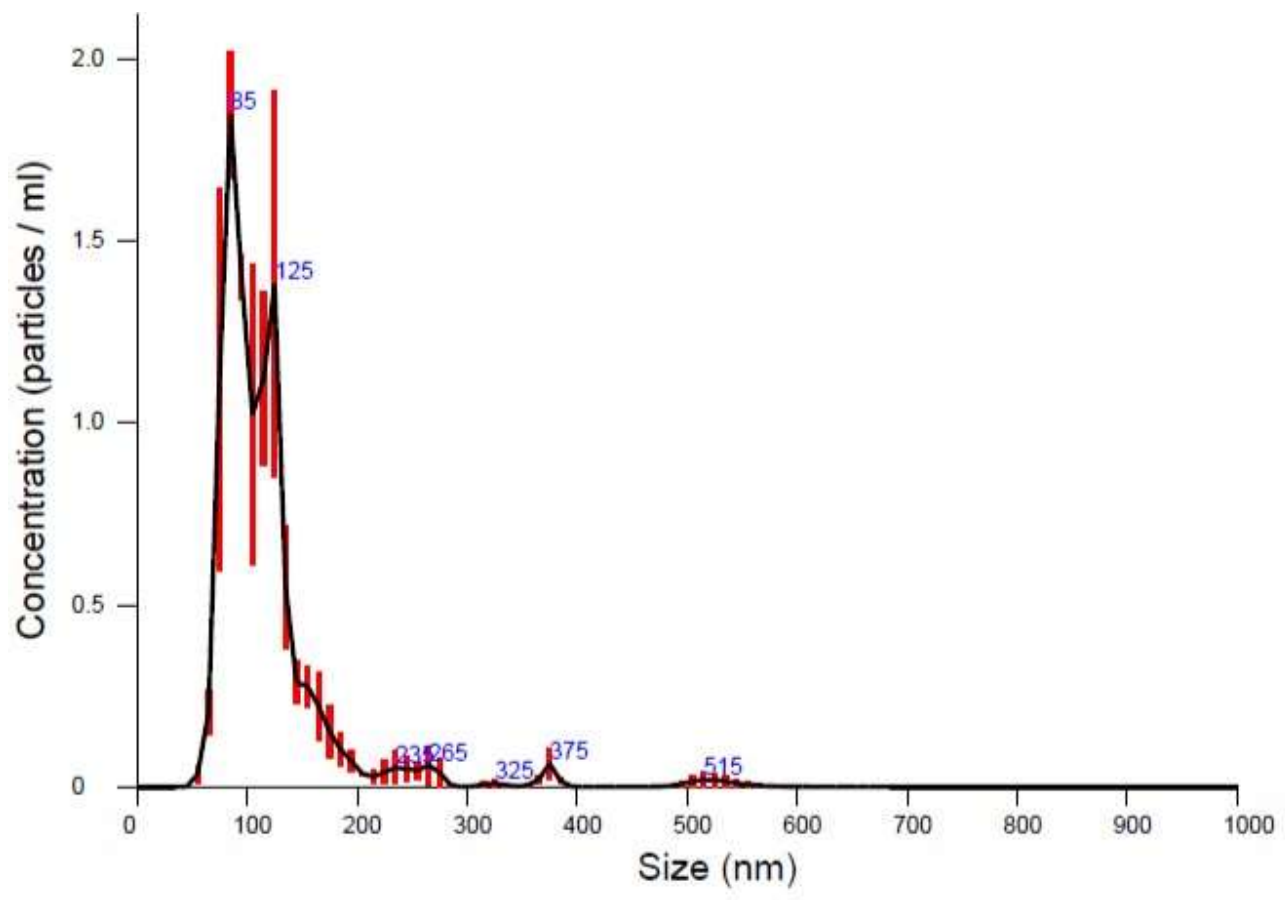

B

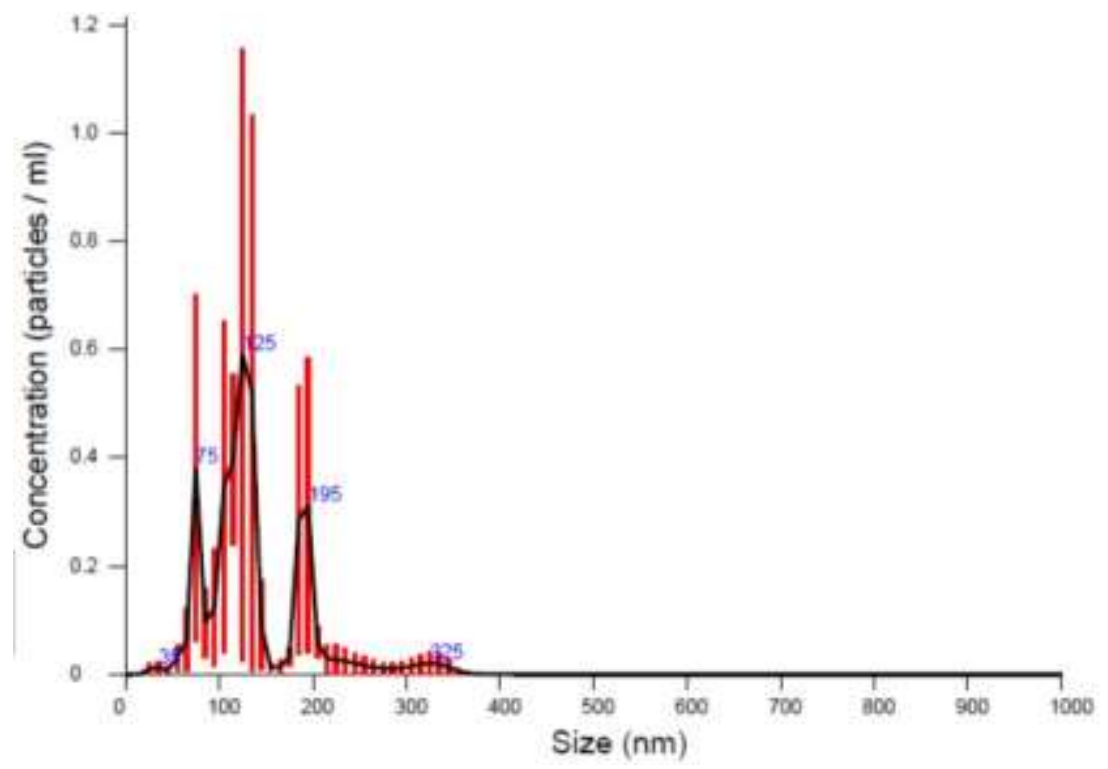


C

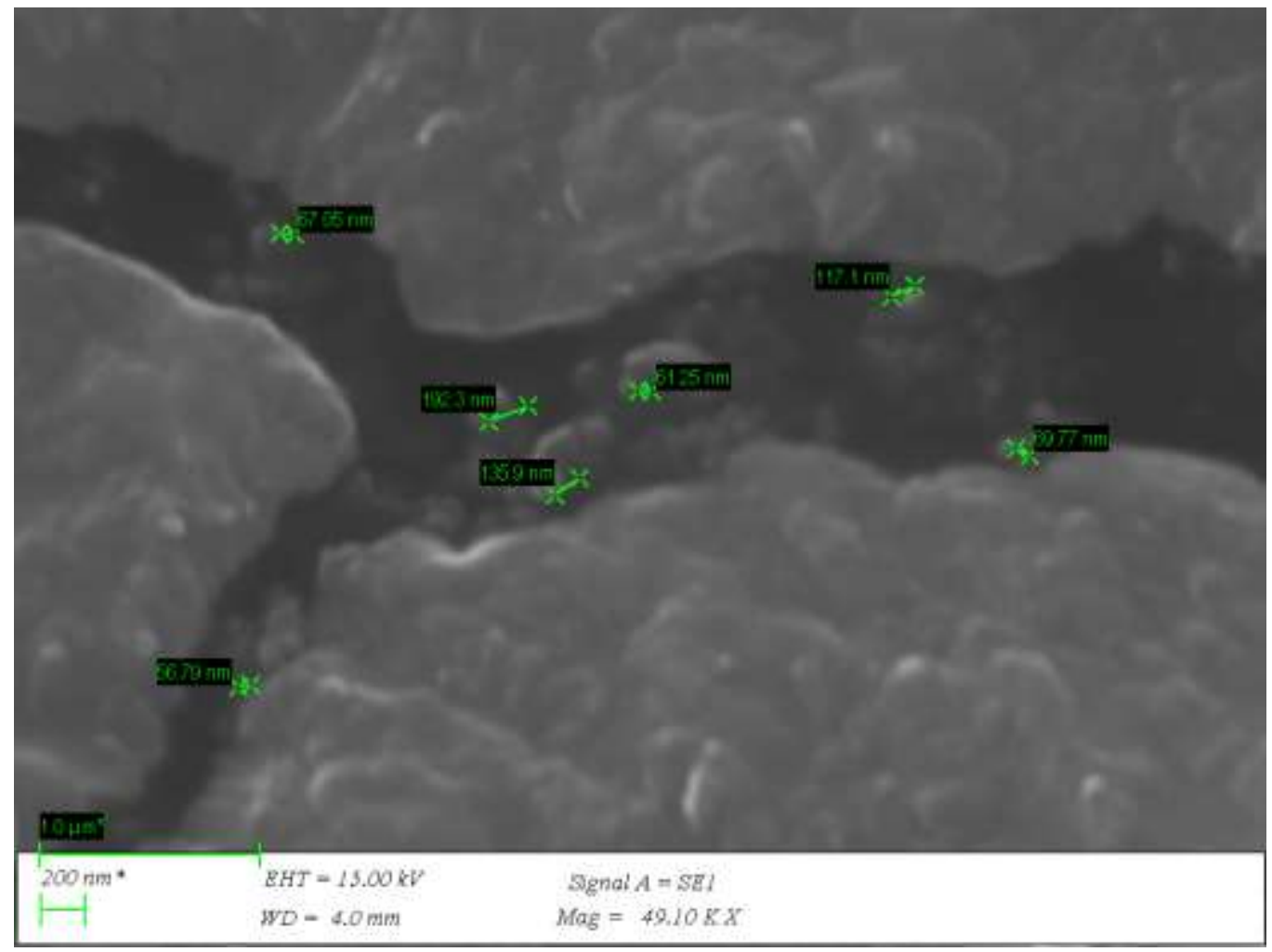

ICBES 118-3 
$\mathrm{D}$

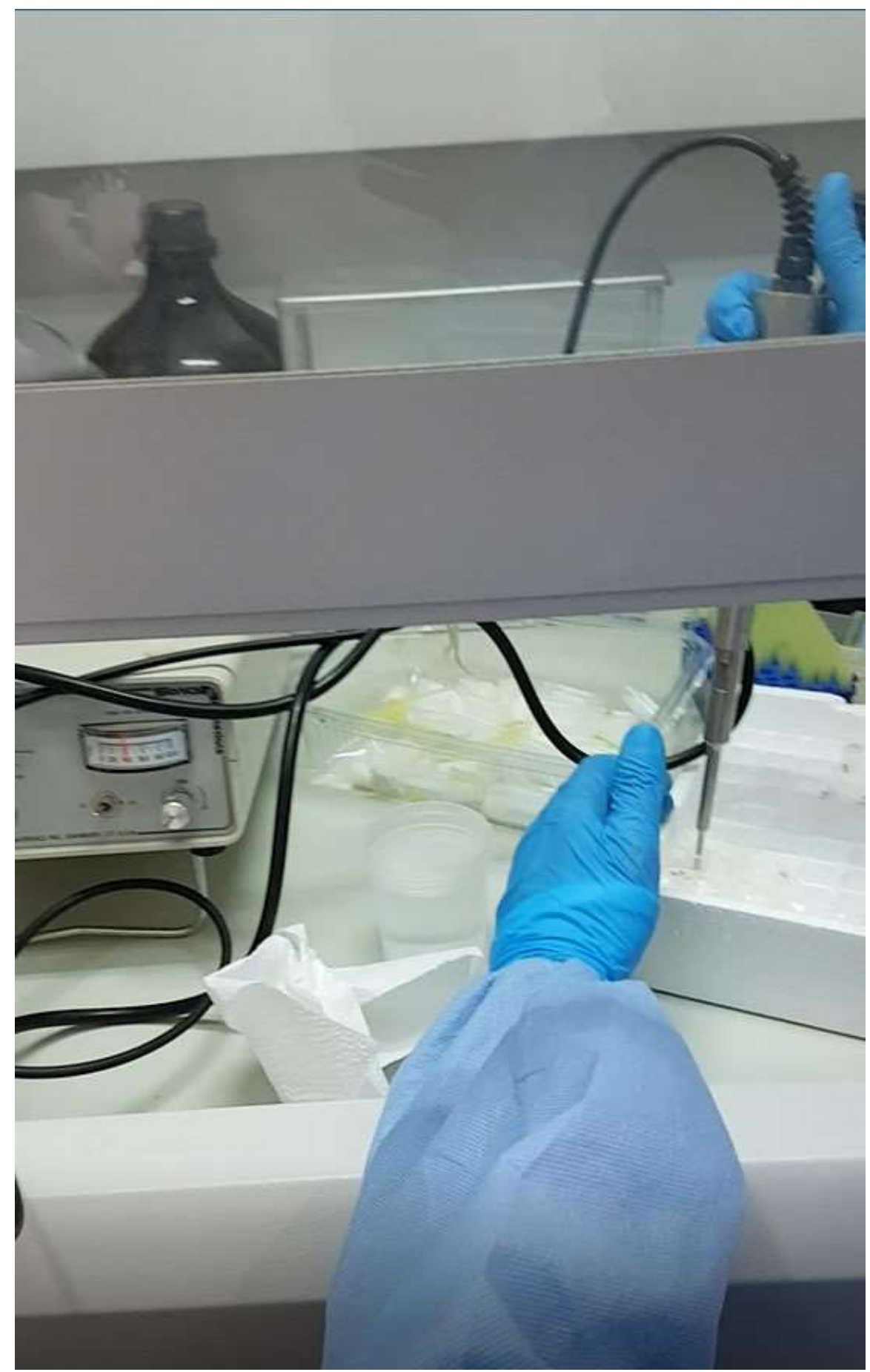

Fig. 1: Empty exosomes of A549 ATCC® CCL-185TM non-small lung cancer cell line before DOX loading was measured A) $127 \mathrm{~nm}$ was detected with impurities $140 \mathrm{~nm}$ from just B) PBS; on the contrary, size intensity was measured as bigger by Malvern Nano/Sizer thereof agglomeration. Size difference detected by these two method, has not been seen during the dexosome size measurements of JAWSII during production. It was ascribed dexosomes of JAWSII has been produced more purely. D) Ultrasonication procedure was just performed to gain max synthetic drug loading to JAWSII dexosomes. 


\section{Conclusion}

In this talk summarizing challenges across production, isolation and antineoplastic drug loading of exosomes. Especially, ultracentrifuge conditions, exosome membrane staining for obtaining images confocal images, cytotoxicity and drug loading efficiency will be discussed. Confocal microscopy has been more convenient way in order to obtain images of exosomes than SEM. High electron beam generally disrupt exosome membrane structure after $70{ }^{\circ} \mathrm{C}$.

\section{Acknowledgements}

Dr. Esra Cansever Mutlu thanks to Bolu Abant Izzet Baysal University through BAP Project 2018.31.01.1359 for financing this research; as well as, appreciation to DEVARGE (http://www.deva.com.tr/tr/devarge) for their donation which provided Doxorubicin antineoplastic agent during experiments. Also Thanks to Prof. Matthew Wood and his group at Oxford University; Especially, Dr. Imre Mager who performed NTA analysis.

\section{References}

[1] S. C. Jang et al., "Bioinspired exosome-mimetic nanovesicles for targeted delivery of chemotherapeutics to malignant tumors," ACS nano, vol. 7, no. 9, pp. 7698-7710, 2013.

[2] P. Vader, X. O. Breakefield, and M. J. Wood, "Extracellular vesicles: emerging targets for cancer therapy," Trends in molecular medicine, vol. 20, no. 7, pp. 385-393, 2014.

[3] S. E. Andaloussi, I. Mäger, X. O. Breakefield, and M. J. Wood, "Extracellular vesicles: biology and emerging therapeutic opportunities," Nature reviews Drug discovery, vol. 12, no. 5, p. 347, 2013.

[4] T. Baranyai et al., "Isolation of exosomes from blood plasma: qualitative and quantitative comparison of ultracentrifugation and size exclusion chromatography methods," PLoS One, vol. 10, no. 12, p. e0145686, 2015.

[5] M. S. Kim et al., "Development of exosome-encapsulated paclitaxel to overcome MDR in cancer cells," Nanomedicine: Nanotechnology, Biology and Medicine, vol. 12, no. 3, pp. 655-664, 2016.

[6] X. Jiang, C. Shen, J. Rey-Ladino, H. Yu, and R. C. Brunham, "Characterization of murine dendritic cell line JAWS II and primary bone marrow-derived dendritic cells in Chlamydia muridarum antigen presentation and induction of protective immunity," Infection and immunity, vol. 76, no. 6, pp. 2392-2401, 2008.

[7] E. Cansever Mutlu, O. Kaya, A. Birinci Yildirim, and A. Cetinkaya, "Exosome Production, Isolation and Characterization from A549 Epithelial Carcinoma Cells," Hacettepe J. Biol. \& Chem, vol. Article in press, 2019. 